\title{
IUFOST2006/547 \\ Fructans reduced the severity of colitis induced by dextran sodium sulphate in mice as a preventive intervention but not as a curative treatment
}

\author{
C. Hoebler, E. Gaudier, J. Maisonneuve and M. Rival \\ Human Nutrition Research Center, BP 71627, 44316 Nantes, France \\ hoebler@nantes.inra.fr
}

Inulin and fructo-oligosaccharides (FOS) are known to be fermented in the colon, producing high level of butyrate and stimulating the growth of lactate-producing bacteria. The butyrate-producing fermentation is suggested to stimulate the trophicity of the intestinal mucosa and maintain the integrity of the non-immune intestinal barrier. The mucus layer, lining epithelial cells, is suggested to contribute to the maintain of intestinal barrier. The aim of the present study was to investigate whether, a mixture of inulin and FOS (IF: Synergy1, Orafti) can protect the colonic mucosa from inflammatory damages or restore its integrity in established colitis, by modulating mucin production.

Two separate experiments tested the preventive and curative properties of IF (4\%) versus basal diet (BD) in dextran-sodium sulfate (DSS)-induced colitis in Balb/c mice. In the preventive conditions, mice received BD or IF during 21 days. From day 12, they also received 1.3\% DSS for 9 days in drinking water. In the curative conditions, mice first recieved $1.3 \%$ DSS alone for 7 days and for the last 2 weeks received $0.2 \%$ DSS and BD or IF. In both experiments, DSS-treated mice were compared to healthy mice fed basal diet (BD) or supplemented IF diet (IF). We investigated fermentation parameters (cecal pH, short chain fatty acid [SCFA] concentration), inflammatory damages (macroscopic mucosal score, diarrhoea, MPO activity), and mucin barrier (Muc gene expression).

In the preventive conditions, IF fermentation increased the SCFA production only in proximal colon of DSStreated mice $(\mathrm{P} \leq 0.05)$. IF diet reduced the inflammation and lowered MPO activity $(\mathrm{P}<0.05)$ of colonic tissue, without changing the macroscopic inflammation parameters. The significant $(\mathrm{P} \leq 0.05)$ increase of the expression of Muc genes (Muc2, Muc3, Muc4) induced by DSS treatment was not modulated by IF diet. In the curative conditions, IF diet stimulated the fermentation in distal colon of DSS-treated mice, but DSS increased the SCFA concentration in all caeco-colonic segements of DSS-treated mice with comparison to control mice $(\mathrm{p} \leq 0.05)$. However, IF diet did not modify any inflammation parameter. Only Muc 4 gene expression was changed by the injuries of mucosal induced by DSS treatment.

A diet supplementated with a nutritional level of IF (4\%), given in preventive conditions, reduced the severity of DSS-induced colitis, but failed in restoring the integrity of ceco-colic mucosa in already established colitis. Mucins did not seem involved in the preventive effect of IF diet. These results confirm the potential efficacy of some dietary fibre in preventing inflammatory bowel disease. 